\title{
THE ESSENCE OF TRADITIONAL CHINESE FURNITURE
}

\author{
APPLIED EXPANSIVE RESEARCH ON TENDON-AND-MORTISE STRUCTURE \\ IN MODERN FURNITURE DESIGN \\ ${ }^{1}$ Jie WANG, ${ }^{2}$ Jun HAN \\ ${ }^{1}$ Breuer Marcel Doctoral School, Faculty of Engineering and Information Technology, \\ University of Pécs, Boszorkány u. 2. 7624 Pécs, Hungary \\ e-mail:34244119@qq.com and \\ ${ }^{1,2}$ College of Art and Design, Inner Mongolia University of Science and Technology
}

Received 7 January 2016; accepted 27 April 2016

\begin{abstract}
Mortise and tenon structure is the essence of traditional Chinese creations, as the main structure of traditional Chinese furniture has its historical origins, fitting in with the background and aesthetic standards of the time. This paper explores the possibility for a new understanding and re-discovery of its use and artistic value in the context of modern times.
\end{abstract}

Keywords: Furniture, Mortise and tenon structure, Modular furniture design, Material, Decorative function

\section{Introduction}

Being part of the essence of traditional Chinese creations, mortise and tenon is central to wood structure (Fig. 1, [1, pp. 8]); it has been present in our life from ancient times up to now, in large palace or temple building groups, in small household items, furniture or tools, just to mention a few examples. It features a form of timber frame connected to another. A node technology is applied on the basis of wood property. In so-called tenon, a convex-shaped object or piece fits within the concave part of another while the mortise object is the hole in a tenon, which is concave [2, pp. 1].

Instead of existing alone, mortise and tenon is closely related to furniture production (Fig. 2, [2, pp. 41]). It takes on the basic quality of traditional Chinese furniture: exquisite, beautiful and practical. This concave and convex model has exerted a wider impact on furniture production around the world [3]. In the era of modern, fast, diverse and simple life style, people's ideas about following the trend of the times became diversified. Mortise and tenon as the main structure of traditional furniture has its 
historical origins, and also fits the background and aesthetic standards of our time. As few things exist independently, for its own sake, it cannot put aside the furniture design and only talk about mortise and tenon itself; the furniture always has close relationship with the way of life and the habits, behavior of people, that is to say research on the mortise and tenon must start from understanding the whole context of the concept. It is of vital importance to think about the life style, aesthetic ideas and needs of modern people [4, pp. 173-180]. This paper explores the possibilities of the use and artistic value of mortise and tenon in the context of modern time and accordingly leads to three conclusions.

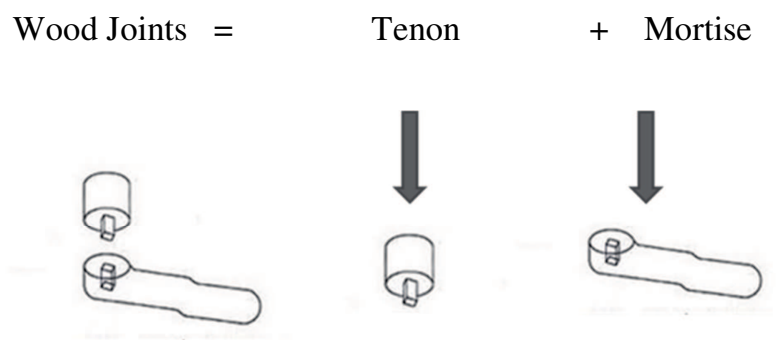

Fig. 1. Mortise and tenon structure [1, pp. 8]
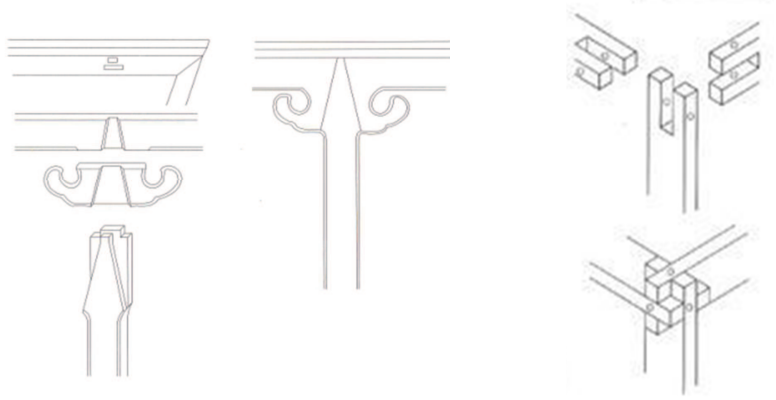

Fig. 2. Mortise and tenon structure is the part of furniture [2, pp. 41]

\section{First, the detachable mortise and tenon structure and modular furniture design}

Modular furniture design refers to the functional analysis and judgment of the furniture components. Modular furniture production is to process components into standardized functional modules, through the selection and combination of modules to design different furniture styles so as to adapt to mechanical or even automated production. Independent unit or interface of modular furniture is usually typical and universal, the core of the modular structure is able to easily exchange module, is 
conducive to transform the function and generate a new derivative products, which are very important in modern furniture design. Modular characteristics are as follows [5, pp. 18-26]:

1. Standardization: Standardization is the main feature of the module. Modular furniture also has other features, which as flexible combination, detachability and reusability, the modules of modular furniture can be detached and combined with each other in several ways, in case parts of the unit components would be damaged or broken, they can be partially replaced, rather than all thrown away, so that the use of furniture products can be easily continued and this realizes sustainability. This requires that the structure of the modular furniture is not a fixed structure, but can be a flexible, detached structure. To do this, the furniture structure must be standardized; all interfaces of modular furniture must be combined with the universal mortise and tenon structure in order to achieve universality, so as to ensure the modular furniture's feature of flexible combination;

2. Combination: Combination is one of the basic characteristics of the modular furniture. The modular product is composed of different modules, and the standard module interface is the base of the combination of modules. Without the combination of modules, modules cannot be integrated into a product with specific function;

3. Versatility: It is another basic feature of modular furniture. And its precondition is the character of interchangeability, it means that the parts of the production manufactured at different times or different locations can be assembled or replaced optionally without modification. Module as a universal component, it is based on the standardization of the interface, which enables the module to be used in different products and ensure the integrity function and usage. The higher complexity of the product, the more prominent is its superiority.

Through the research on the theory and the actual project of the modular furniture design, not only the $32 \mathrm{~mm}$ furniture system but also modular furniture manufacture with the key of unified interface and unified module in order to achieve modular furniture product [1, pp. 46-51]. Through the study it can be found that detachable mortise and tenon structure is in line with modern furniture modular design concept (Fig. 3, [6]). As the structure is easy to detach, so it is easy to maintain, suitable for recycling, and can be developed as modular production. Therefore, it is feasible to create a modular furniture design by devising a mortise and tenon structure as the core of a unified connection.

In the current state of fast-paced life, people want their lives easier, more convenient, with more experience, therefore, use the modular design concept, research on the mortise and tenon structure design, exploring the possibility of expanding furniture design [7]. The author in line with the modern modular furniture design, deconstructs and restructures the mortise and tenon structure according to the design concept of being easy to detach. In the modern furniture design by extracting mortise and tenon structure, simplifying it and combining it with modular furniture design concept, it is possible to explore the development of mortise and tenon structure into modern modular furniture design. Mortise and tenon is of a various and complex 
structure. Modern modular furniture design needs the simplification and modification of the original structure in order to achieve universality. Based on the new form of single module structure, furniture can be changed into different forms, reflecting the characteristics of variety. According to their own needs, users can purchase different numbers of single modules and assemble them by themselves, so that the function and space of furniture usage will have more possibilities, to maximize the function and expand the space that furniture can be used in [8, pp. 389-393].
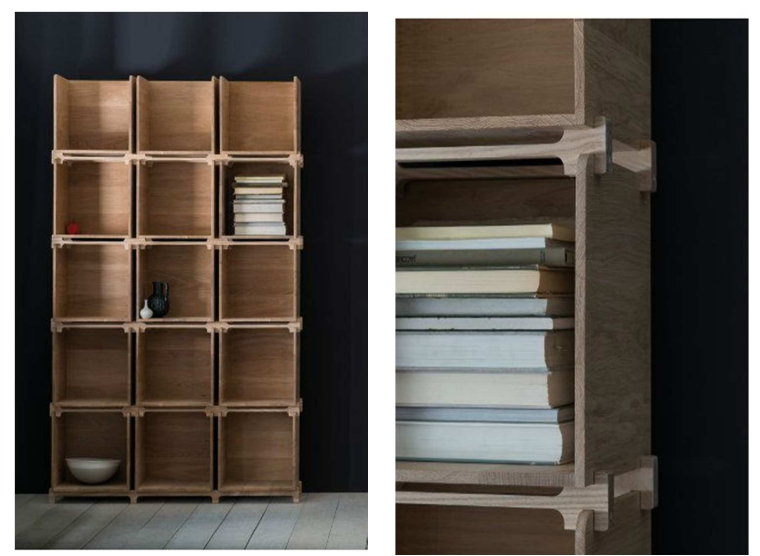

Fig. 3. Detachable mortise and tenon structure and modular furniture [6]

\section{Second, mortise and tenon structure and new materials, new technology}

In mortise and tenon structure of the traditional furniture precious hardwood with beautiful color and solid texture is used to provide a viable precondition for the manufacture of mortise and tenon frames of various functions. Mortise and tenon structure uses timber carrier to undertake the various components of furniture, which itself has become a part of the furniture [9, pp. 353-357]. But nowadays hardwood has become less and less available, so it is no longer suitable for the development of modern mortise and tenon structure furniture design, because of the shortening of natural resources, hardwood has become very precious, it needs hundreds of years to grow, and excessive cutting will cause environmental problems, so the industry needs to try to use some other materials without damaging the environment too much [10, pp. 151-161]. Thanks to the development of industrialization in modern design, furniture is made of a large number of composite plates, metal materials, acrylic and other new materials by injecting a variety of design elements into modern furniture design with a broader choice [11]. In modern mortise and tenon structure furniture design should try to use new materials to replace the traditional hardwood material (Fig. 4, [12]). In certain circumstances rational use of new materials can solve a lot of problems caused by using traditional mortise and tenon structure [13]. For example, using mortise and tenon structure in the design of small shape, traditional hardwood will have some problems, 
since the structure is too small, the wood will not reach up to the mechanical standard so it might be broken; nowadays it can use some new materials with good mechanical properties to replace the traditional wooden mortise and tenon structure, and through this way, that cannot only realize the design of mortise and tenon structure, but also optimize mortise and tenon structure, solving the problems that existed in the traditional mortise and tenon structure. In a word, the relationship between modern mortise and tenon structure design and materials are mutual stimulation and promotion. People will have different opinions on new materials with changes of times and consciousness, so as to promote the improvement and development of materials. The advantages of new materials not only have a single function, but have more good features under certain conditions, that as good processing properties, texture and appearance quality, economy, strength, surface decoration, environmental protection and so on, so when new materials are used, one should consider the advantages of each material, make full use of different materials and optimize the combination of materials. Material mixture and match were embodied well in traditional mortise and tenon structure furniture design; this design concept can be flexibly used in modern mortise and tenon structure furniture design. Material mixture can improve the decoration and functionality of products, that as some metal or plastic furniture use mortise and tenon structure or some similar structure, or in the traditional mortise and tenon structure it can mix hardwood with other materials. The rational use of new materials for innovative design is essential for the development of modern mortise and tenon structure furniture design to meet the development needs of the times. On the basis of understanding new materials one might make bold attempt and reasonable application to further develop traditional Chinese mortise and tenon structure through modern technology.
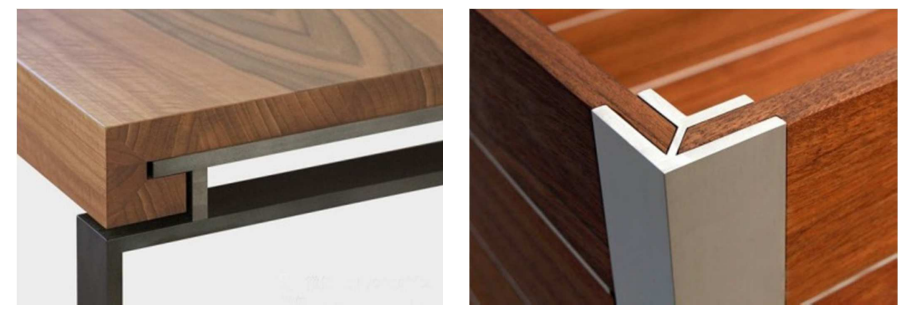

Fig. 4. Wood and metal mortise and tenon structure [12]

In the next place is the application of new technology, in today's era of mass production, traditional handicraft techniques of traditional mortise and tenon structure are no longer able to meet the needs of the modern furniture manufacturing, so the application of new technology is very important, the use of new technology can improve production efficiency, reduce production costs, and is easy to master. The traditional processing of mortise and tenon structure happened mainly by hand, that needed skilled workers who are difficult to hire and also need to be paid high salary, so the process of modern mortise and tenon structure should mainly utilize mechanization, and innovation by using the new technology on the basis of retaining tradition, through this way it can reduce employment costs, because ordinary workers can be quickly trained to undertake the work. Therefore, reasonable application of new technology has a very 
important role for the development of modern mortise and tenon structure furniture design, as it can improve the competitiveness on the market [14, pp. 47-51].

New materials as well as new technology may be utilized to improve and re-design mortise and tenon structure, which eventually facilitates modern furniture design to meet the requirements of mass production, not only considering the materials and technology, but also combining with current local conditions, taking advantage of surface effects, physical properties, structural strength and other aspects to carry out integration and re-create and explore new materials and new technology that adapt to the mortise and tenon structure.

\section{Third, decorative function of mortise and tenon structure}

Traditional mortise and tenon not only has structural function, but also plays a decorative role, setting both practical and aesthetic qualities. The author views it necessary to take advantage of this feature to highlight the design. Designers are expected to break through traditional mortise and tenon mode by converting inner forms into the exposed, thus giving full consideration to the structure traces at the joints of mortise and tenon. The new method thus highlights structure aesthetics beyond visual values, blending function with decorative glamour of the mortise and tenon. This form of mortise and tenon structure has both mechanical property and aesthetic expression, making the structure and form to achieve the perfect unity, in addition, this kind of exposed structure form reflects better the features of identification and detachment [15, pp. 18].

Furniture is not only a simple functional product, but also a widely popular public art. The decoration of the furniture is not only embodied by the external form of the product, but more importantly it will be determined by its internal structure, which because the form of the furniture product is given by the product's structure and the bonding methods (Fig. 5, [16]). For example, frame type mortise and tenon furniture fully reflects the line of decorative arts, has not only the corresponding function, but can also play the role of embellishment, especially the exposed mortise and tenon structure can fill products with natural beauty style that is a manifestation of the beauty of technology. Breaking through inner forms of traditional mortise and tenon structure into new exposed structure to highlight the significant value of the visual aesthetic, this kind of ingenious exposed structure make furniture easier to identify and operate, detached conveniently through the perfect combination of art and technology, to reflect the new spirit of times that integrate traditional culture with modern design aesthetics, more in line with the modern aesthetic concepts and adapt to the modern fast way of life.

The mortise and tenon structure is an integral part of the traditional furniture, and a symbol of Chinese furniture, but the requirements of modern furniture for the mortise and tenon structure is not so high as to fully utilize the characteristics of the material itself and reduce costs by reducing the use of some structural components [17, pp. 155-168]. For example, the mortise and tenon structure will be more efficient by simplifying its form or combining some metal parts with it. Some particularly complex mortise and tenon structures are rarely used in modern furniture design, so there is not much meaning just to solve structural problems by using mortise and tenon structure, 
people have turned to the structure and formal beauty of mortise and tenon, focusing on decoration.
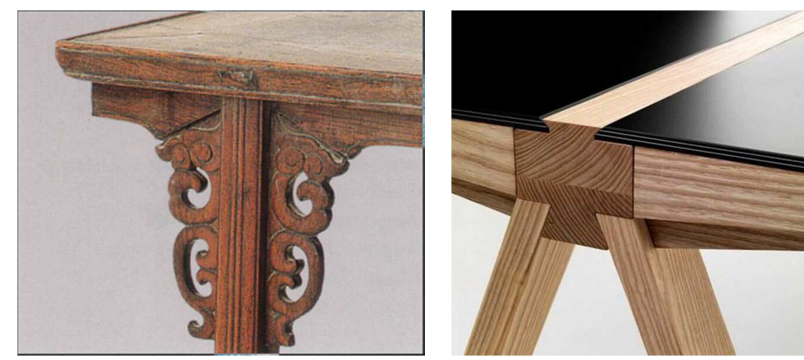

Fig. 5. Decorative function of mortise and tenon structure [16]

\section{Conclusion}

The simple concave-convex relationship carries a profound artistic conception of design and cultural connotation while the mortise and tenon structure is explored from quiet and far deep historical background and beyond [18, pp. 70-73]. Today, in the vigorous development of modern furniture production, it is important to review and carry on the traditional culture and absorb its creams. Mortise and tenon art is of great significance as concave-convex pattern not only inherits the tradition, but leads us thinking about the future development. Through the study of mortise and tenon structure in modern furniture design, it can be combined with modular furniture design, use more modern materials to highlight its decorative force, making it more in line with the modern aesthetic concept, and adapting to modern pace of life, so as to realize the unity between the beauty of technology and art, and reflecting the new spirit which incorporates traditional culture with aesthetics in modern design.

\section{References}

[1] Li S. X. Applied research of mortise and tenon structure in modern hardwood furniture, Master's Thesis, China Academy of Art, 2013.

[2] Wan Q. The research and design of mortise and tenon structure of hardwood furniture, Master's Thesis, Beijing Institute of Technology, 2015.

[3] Austere S. H. Luminosity of Chinese classical furniture, California, University of California Press, 2001.

[4] Portschy Sz. Design partnership between community-engaged architecture and academic education programs, Polalck Periodica, Vol. 10, No. 1, 2015, pp. 173-180.

[5] Sun Y. Y. Applied research of DIY concept in the modular furniture design, Master's Thesis, Jiangnan University, 2007.

[6] http://mp.weixin.qq.com/s?_biz=MzA5MTIyMTQZA==\&mid=200216614\&idx=1\&sn= d0089e29ff89b0a5e154c23e8a38723c\&3rd=MzA3MDU4NTYzMw==\&scene=6\#rd （last visited 10 December 2015). 
[7] Yu, De-hua. Research on human factors engineering of Chinese traditional armchair with four protruding ends, In: The 19th International Conference on Industrial Engineering and Engineering Management (IE\&EM 2012), Qi, Ershi; Shen, Jiang; Dou, Runliang (Eds.) Chansha, China, 2012, Springer, 2013, pp. 13-21.

[8] Xu J. F. On the creation and design methods of new Chinese furniture, Proceedings of 2011 IEEE 12th International Conference on Computer-Aided Industrial Design \& Conceptual Design, Vol. 1, Chongqing, China, 27-29 Nov 2011, pp. 389-393.

[9] Zou W. H. The concept of green design in traditional Chinese furniture, Proceedings of 2012 International Conference on Innovation and Information Management (ICIIM 2012), Vol. 36, 2012, pp. 353-357.

[10] Kiss I., Reith A. Architectural and urban design tools for reducing energy consumption in cities, Polalck Periodica, Vol. 8, No. 3, 2013, pp. 151-161.

[11] Liu X. Y., Timar M. C., Varodi A. M. A preliminary study of three finishing materials for traditional Chinese furniture, http://www.scirp.org/journal/ampc, May 2014 in SciRes, (last visited 10 December 2015).

[12] http://blog.sina.com.cn/s/blog_7df7990a0101jgyb.html (last visited 15 October 2015).

[13] Byars M. 50 tables innovations in design and materials, Introduction by Sylvain Dubuisson, Published by Rotovision, 1996.

[14] Wei H. L. Applied research of the design concept of Ming style furniture in modern wood products design, Master's Thesis, Qiqihar University, 2012.

[15] Deng N. The beauty of mortise and tenon structure, Master's Thesis, Xi'an Academy of Fine Arts, 2013

[16] http://www.mytater.com/post/25105/ (last visited 30 October 2015).

[17] Dalvand M., Ebrahimi G., Haftkhani A. R., Maleki S. Analysis of factors affecting diagonal tension and compression capacity of corner joints in furniture frames fabricated with dovetail key, Journal of Forestry Research, Vol. 24, No. 1, 2013, 155-168.

[18] Lin Y. The foreign Ming-style furniture fascination, China Today, 10 June 2015. 